\title{
ESÔFAGO DE BARRETT: novos métodos diagnósticos
}

DESCRITORES - Esôfago de Barrett, diagnóstico.

O esôfago de Barrett (EB) é hoje definido como a substituição do epitélio escamoso do esôfago distal por epitélio colunar, principalmente na presença de epitélio colunar especializado, com presença de metaplasia intestinal (MI) ${ }^{(1)}$. Portanto, seu diagnóstico é realizado através de biopsias colhidas por endoscopia, condicionando sua acuidade à capacidade do endoscopista e da tecnologia empregada. Tratando-se de um dos poucos precursores identificáveis do adenocarcinoma do esôfago, novas técnicas que procuram melhor diagnóstico endoscópico do EB devem propiciar maior sensibilidade tanto na confirmação do epitélio especializado, quanto na identificação de áreas suspeitas, dirigindo, assim, as biopsias a serem realizadas $^{(2)}$.

Na procura de melhor acurácia para avaliação endoscópica do esôfago de Barrett, vem sendo muito utilizada a cromoscopia, associada ou não com novas tecnologias, como a endoscopia com magnificação de imagens ${ }^{(10)}$. Vários corantes têm sido utilizados, como o índigo de carmim (corante não absorvido pelas células), usado para analisar a arquitetura do epitélio colunar e dirigir a biopsia na suspeita de neoplasias localizadas em áreas com MI; a coloração do epitélio escamoso com lugol a $2 \%$, para identificar e localizar irregularidades na linha de junção escamocolunar nos segmentos curtos do EB; a violeta cristal ou a violeta de cresyl para marcar também a superfície do epitélio colunar; mais recentemente, com grande ênfase, a utilização do azul de metileno com afinidade específica para o epitélio absortivo com bordas em escova e células caliciformes ${ }^{(9)}$. O azul de metileno, descrito originalmente no Japão em 1979 para pesquisa de MI em epitélio gástrico, passou a ser utilizado no EB a partir de 1996, por CANTO et al.(3), que descreveram a coloração pelo azul de metileno como focal ou difusa, correlacionando a última com extensão maior de epitélio colunar especializado, geralmente presente nos segmentos longos de EB. Já a coloração focal estaria relacionada à presença do epitélio especializado em apenas algumas áreas, como no EB curto, onde este se mistura com a metaplasia gástrica ${ }^{(5)}$.
A coloração também pode variar conforme a intensidade e a heterogeneidade. Os segmentos longos de EB, sem displasia, coramse de azul escuro, de forma homogênea, uma vez que a maior parte do epitélio é do tipo intestinal e o corante é absorvido pelo citoplasma das células colunares e caliciformes. Já na displasia de alto grau ou no adenocarcinoma, pelo aumento do núcleo e diminuição do citoplasma, com menor presença ou mesmo ausência de células caliciformes, a coloração com azul de metileno é focal e de pouca intensidade (azul claro), ou mesmo ausente. Portanto, a coloração anormal do epitélio colunar pode ser usada para localizar ou dirigir as biopsias na busca de áreas de displasia ou adenocarcinoma ${ }^{(4)}$.

CANTO et al. ${ }^{(3)}$ mostraram grande acurácia do método, utilizando videoendoscópios convencionais. Obtiveram resultados reprodutíveis após 4 semanas, sensibilidade de $95 \%$ e especificidade de $97 \%$, com acurácia de até $90 \%$ para detecção de displasia de alto grau, quando comparada com o estudo histológico ${ }^{(5)}$. Mostraram, porém, coloração de até $42 \%$ dos casos no grupo-controle, incluindo em áreas sem MI.

Em outro estudo, KIESSLICH et al. ${ }^{(8)}$ confirmaram alta sensibilidade para o azul de metileno (98\%) entretanto, com especificidade muito menor (61\%). Não observaram contribuição do azul de metileno na detecção de quatro pacientes da casuística com adenocarcinoma, uma vez que essas áreas haviam sido identificadas antes mesmo da cromoscopia.

Alguns estudos conflitantes quanto ao real benefício do azul de metileno foram recentemente publicados. Comparando áreas coradas e não-coradas em apenas nove pacientes com EB, DAVE et al. ${ }^{(6)}$ mostraram correlação muito menor entre áreas coradas e a presença de epitélio com MI (sensibilidade de $57 \%$ e especificidade de $32 \%$ ), e WO et al. ${ }^{(13)}$ observaram sensibilidade de $53 \%$ e especificidade de $51 \%$. Nesses estudos também não foi comprovado o papel da coloração com azul de metileno para melhor avaliar as áreas de displasia de alto grau ou adenocarcinoma. O estudo de SAPORITI et al. ${ }^{(11)}$, publicado neste número dos ARQUIVOS de GASTROENTEROLOGIA, mostrou que o emprego da cromoscopia com azul de 
metileno aumenta significativamente o tempo do exame endoscópico, além de não apresentar vantagens para o diagnóstico do EB.

Novas perspectivas no diagnóstico e seguimento do EB vêm surgindo recentemente com a utilização da cromoscopia associada a exames endoscópicos com magnificação de imagem. GUELRUD et al. ${ }^{(7)}$, utilizando magnificação com aumento de 35 vezes e coloração com ácido acético diluído a 1,5\%, classificaram a metaplasia colunar em quatro padrões bem estabelecidos. Um dos padrões (tipo IV aspecto ceribriforme) se relacionou em $100 \%$ dos casos, com a presença de MI. A correlação final demonstrou sensibilidade de 1,5\% para detecção de MI com endoscopia convencional, 8,5\%, quando se utilizou coloração com ácido acético, $38 \%$, apenas com magnificação de imagem e $100 \%$ com a associação da magnificação e coloração do ácido acético. Ainda faltam estudos para verificar a eficácia deste método na detecção de padrões que representem áreas ocultas de neoplasia ou displasia no EB.

Outras modalidades vêm sendo pesquisadas no estudo das doenças malignas do aparelho digestivo, como a espectroscopia e a fluoroscopia. A primeira se baseia na absorção pelo tecido tumoral de substâncias exógenas (fluoróforas), que reagem com nucleotídios nucleares das células, administradas antes do exame de espec- troscopia. Este é realizado através de endoscopia onde são emitidos fótons por um "probe" na superfície mucosa do esôfago. A absorção e refração desses fótons seria diferente, dependendo da absorção dos fluoróforos em cada tecido, podendo mostrar, inclusive, a evolução do tecido de displasia de baixo grau para de alto grau ou mesmo adenocarcinoma. A fluoroscopia também utilizada no sentido de identificar áreas de displasia de alto grau, mostrando em estudo recente, sensibilidade e especificidade de $87 \%{ }^{(9)}$. Apesar destas novas perspectivas, os dois métodos apresentam-se como opções de alto custo, dependendo de outros especialistas, como físicos e técnicos para manuseio dos aparelhos ${ }^{(12)}$.

A pesquisa no campo da ciência óptica certamente vai trazer desenvolvimento nas imagens endoscópicas, permitindo a realização de biopsias em lesões cada vez mais precoces. Seu desenvolvimento caminha em dois sentidos: na magnificação de imagens e na espectroscopia. Sem dúvida, a magnificação de imagem apresenta vantagens quanto à capacidade de dirigir as biopsias, principalmente pela simplicidade relativa de sua execução, sem utilização de acessórios muito sofisticados.
Ivan CECCONELLO* Sergio SZACHNOWICZ*

Cecconello I, Szachnowicz S. Barrett's esophagus: new diagnostic tools. Arq Gastroenterol 2003;40(3): 137-138

HEADINGS - Barrett esophagus, diagnosis

\section{REFERÊNCIAS BIBLIOGRÁFICAS}

1. Appelman HD. Is the presence of specialized epithelium necessary for the diagnosis of Barrett's esophagus? In: Giuli R, Tytgat GNJ, DeMeester TR, Galmiche JP, editors. O.E.S.O. The esophageal mucosa. Amsterdam: Elsevier Science; 1994. p.878-9.

2. Bruno MJ. Magnification endoscopy, high resolution endoscopy, and chromoscopy; towards a better optical diagnosis. Gut 2003;52 Suppl 4:iv7-11.

3. Canto MI, Setrakian S, Petras RE, Blades E, Chak A, Sivak MV Jr. Methilene blue selectively stains intestinal metaplasia in Barrett's esophagus. Gastrointest Endosc 1996;44:1-7.

4. Canto MI, Setrakian S, Willis JE, Chak A, Petras RE, Sivak MV. Methilene blue staining of dysplastic and nondysplastic Barrett's esophagus: an in vivo and ex vivo study. Endoscopy 2001;33:391-400.

5. Canto MI, Yoshida T, Gossner L. Chromoscopy of intestinal metaplasia in Barrett's esophagus. Endoscopy 2002;34:330-6.

6. Dave U, Shouisa S, Westay D. Methilene blue staining: is it really useful in Barret's esophagus? Gastrointest Endosc 2001;53:333-5.
7. Guelrud M Herrera I, Essenfeld H, Castro J. Enhanced magnification endoscopy: a new technique to identify specialized intestinal metaplasia in Barrett's esophagus. Gastrointest Endosc 2001;53:559-65.

8. Kiesslich R, Hahn M, Herrmann G, Jung M. Screening for specialized columnar epithelium with methylene blue: chromoendoscopy in patients with Barrett's esophagus and a normal control group. Gastrointest Endosc 2001;53:47-52.

9. Lambert R. Diagnosis of esophagogastric tumors. Endoscopy 2002;34:129-38

10. Lambert R, Rey JF, Sankaranarayanan R. Magnification and chromoscopy with the acetic acid test. Endoscopy 2003;35:437-45.

11. Saporiti MRL, Souza RCA, Pisani JC, Amarante HMBS, Carmes ER, Danielle Giacometti Sakamoto DG. Cromoendoscopia com azul de metileno para diagnóstico de esôfago de Barrett. Arq Gastroenterol 2003;40:139-47.

12. Wallace M, van Dam J. Enhanced gastrointestinal diagnosis: light-scattering spectroscopy and optical coherence tomography. Gastrointestinal Endosc Clin N Am 2000;10:71-80.

13. Wo JM, Ray MB, Mayfield-Stokes S, Al-Sabbagh G, Gebrail F, Sloane SP, Wilson MA. Comparasion of methilene blue-directed biopsies and conventional biopsies in the detection of intestinal metaplasia and dysplasia in Barrett's esophagus: a preliminary study. Gastrointest Endosc 2001;54:294-301.

\footnotetext{
* Disciplina de Cirurgia do Aparelho Digestivo, Departamento de Gastroenterologista da Faculdade de Medicina da Universidade de São Paulo.
} 